\title{
Phage Peptides Mediate Precision Base Editing with Focused Targeting Window
}

Kun Jia ${ }^{1,2,4,6}$, Yan-ru Cui ${ }^{1,3,5,6}$, Shisheng Huang ${ }^{3,5}$, Peihong Yu ${ }^{1,3,5}$, Zhengxing Lian ${ }^{4}$, Peixiang Ma ${ }^{1}$, Jia Liu ${ }^{1,3 *}$

${ }^{1}$ Shanghai Institute for Advanced Immunochemical Studies, ShanghaiTech University, Shanghai, 201210, P.

R. China

${ }^{2}$ The State Kay Laboratory of Respiratory Disease, First Affiliated Hospital of Guangzhou Medical University, Guangzhou, 510120, China.

${ }^{3}$ School of Life Science and Technology, ShanghaiTech University, Shanghai, 201210, P. R. China

${ }^{4}$ Beijing Key Laboratory of Animal Genetic Improvement, China Agricultural University, 2 Yuanmingyuan

West Rd., Haidian District, Beijing 100094, China

${ }^{5}$ University of Chinese Academy of Sciences, Beijing, 100049, China

${ }^{6}$ These authors contributed equally to this work.

Correspondence should be addressed to J.L.(liujia@shanghaitech.edu.cn) 
bioRxiv preprint doi: https://doi org/10.1101/202011.02 364430; this version posted November 2,2020 . The copyright holder for this preprint (which was not certified by peer review) is the author/funder, who has granted bioRxiv a license to display the preprint in perpetuity. It is made available under aCC-BY-NC-ND 4.0 International license.

\begin{abstract}
Cytidine base editors (CBE) are novel genome engineering tools that can generate $\mathrm{C}$-to-T nucleotide substitutions without introducing double-stranded breaks (DSBs). Instead of generating single-point mutations, CBEs induce nucleotide substitutions at wobble positions within the 20 -nucleotide target site. A variety of strategies have been developed to improve the targeting scope and window of CBEs. Among these strategies, molecular switches that can temporally control CBE activities represent compelling options. In this study, we investigated the feasibility of using a bacteriophage-derived peptide, referred to as G8P PD, as the off-switch of CBEs. We showed that G8P PD could be employed to control the activities of and improve the targeting window of $\mathrm{A} 3 \mathrm{~A}$ and $\mathrm{BE} 3 \mathrm{CBEs}$ and adenine base editor 7.10 (ABE7.10). Notably, in a cell-based disease model of Marfan syndrome, G8P $P_{P D}$ facilitated A3A CBE-based gene correction with a more focused targeting window and improved the percentage of perfectly edited gene alleles from less than $4 \%$ to more than $38 \%$ of the whole population. Our study presents the first peptide off-switch that can improve the targeting scope of CBEs, thus highlighting the importance of the temporal control of BE activity for precision base editing.
\end{abstract}




\section{Introduction}

Clustered regularly interspaced short palindromic repeats (CRISPR)-CRISPR-associated genes (Cas) is the bacterial adaptive immune system for protecting host organisms from invading pathogens ${ }^{1-3}$. Owing to the modular feature, type II CRISPR systems, particularly CRISPR-Cas9, have been widely used for genome editing, transcriptional and epigenetic modulation and molecular imaging ${ }^{4}$. CRISPR-Cas9 can be directed to human genome by single-guide RNA (sgRNA) to create double-stranded breaks (DSBs) at targeted genomic loci ${ }^{5-8}$. In human cells, DSBs are repaired by two competing DNA repair pathways: error-prone nonhomologous end joining (NHEJ) and homology directed repair (HDR). The latter repair pathway can facilitate CRISPR-Cas9-mediated gene correction of pathogenic mutations ${ }^{4}$.

Recent studies have highlighted base editors (BEs) as an efficient genome editing tool for precision gene therapy $^{9-11}$. BEs are fusion proteins comprising a catalytically inactive Cas nuclease and a nucleobase deaminase ${ }^{12,13}$. Unlike CRISPR-Cas9 genome editing tools, BEs generate precise base substitutions without introducing DSBs, thus avoiding concurrent, competing NHEJ events that incorporate nucleotide insertions and deletions (indels) ${ }^{14}$. Cytidine base editors (CBEs) and adenine base editors (ABEs) have been developed to realize genomic alterations of C-G to T-A and A-T to G-C, respectively. Besides DNA editing, Cas variants with RNA-binding capacities have been adapted for programmable RNA editing ${ }^{15,16}$.

The widely used third-generation CBEs, referred to as BE3, are fusion proteins composed of rat APOBEC (rAPOBEC), Cas9 D10A nickase and uracil glycosylase inhibitor (UGI). BE3 has a five nucleotide targeting window ranging from position 4 to 8 , counting the protospacer adjacent motif (PAM) trinucleotides as positions 21 to $23^{12}$. An engineered CBE variant A3A, where rAPOBEC is replaced with human APOBEC3A (hAPOBEC3A), has a wider targeting window at positions 2 to 13 and is capable of editing methylated genomic regions ${ }^{17}$. Engineering endeavors have been made to improve the editing window of both BE3 (rAPOBEC-nCas9-UGI) ${ }^{18}$ and A3A (hAPOBEC3A-nCas9-UGI) CBEs ${ }^{19}$. Compared with ABEs, CBEs have 
higher genome-wide off-target events ${ }^{20,21}$. Despite of the considerable studies, the origin of the flexible editing windows and genome-wide off-target of CBEs are not completely understood.

It has been well known that excessive dosage of Cas9 and sgRNA can lead to elevated off-target mutations $^{22}$. This finding led to the widespread use of directly delivered Cas9-guide RNA (gRNA) ribonucleoproteins (RNPs), which can improve the genome editing specificity by limiting the exposure of genome to gene-editing agents ${ }^{23}$. Similarly, delivery of BE-gRNA RNP can increase the base editing specificity compared with transfection of BE-coding plasmids ${ }^{24}$. Besides the use of gene-editing agents with short intracellular half time ${ }^{23}$, molecular switches provide compelling opportunities to improve the specificity of CRISPR-Cas via the temporal control of its cellular activity. Indeed, a variety of CRISPR-Cas inhibitors have been developed, including naturally occurring anti-CRISPR (Acr) proteins ${ }^{25-27}$, synthetic oligonucleotides $^{28}$ and small molecules ${ }^{29}$. These inhibitors can improve the genome editing specificity of Cas9 $\mathrm{RNPs}^{30}$ and modulate the cellular activity of BEs ${ }^{29}$.

In a previous study, we have identified bacteriophage-derived peptides G8PD as inhibitors to Streptococcus pyogenes Cas9 (SpCas9) ${ }^{31}$. Herein, we hypothesized that the broad targeting window of CBEs is attributed, at least in part, to the excess intracellular dosage of base editing agents that could be alleviated by timed delivery of G8P $\mathrm{PD}$. Indeed, we found that overexpression of the peptide inhibitors of SpCas9 in human cells could direct BE3 and A3A CBEs to more focused editing windows and facilitate precision gene correction in a cell model of Marfan syndrome ${ }^{32}$. 


\section{Results}

\section{G8PPD peptides inhibit CBE targeting of EGFP reporter}

We have reported in a previous study that the periplasmic domain of the major coat proteins (G8P $\mathrm{PD}_{\text {) from }}$ M13 and $\mathrm{f1}$ bacteriophages (Fig. 1a) can inhibit the in vitro and cellular activities of SpCas $9^{31}$. Because these peptides suppress SpCas9 activity by disrupting SpCas9-sgRNA binding ${ }^{31}$, we hypothesized that they could also act as inhibitors to cytidine base editors (CBEs) the activity of which also rely on sgRNA-guided DNA binding of SpCas9 domain. In order to evaluate the inhibitory activities of M13 and f1 G8P PD on CBEs, we constructed an enhanced green fluorescent protein (EGFP) reporter carrying an inactivating mutation Y66C at the chromophore (Supplementary Fig. 1a). An sgRNA was designed to target the Y66C mutation site for introducing C-to-T conversion by CBE (Supplementary Fig. 1b). This mutation can correct EGFP-Y66C to wild-type genotype, yielding fluorescent cells that can be readily detected by flow cytometry. Inhibition of A3A CBE by G8P PD peptides will lead to reduced EGFP fluorescence (Supplementary Fig. 1a).

Our previous study has shown that G8P PD peptides bind to sgRNA-free SpCas9 (apo-Cas9) and that maximum SpCas9-inhibiting activity of G8P $P_{P D}$ can be achieved by pre-incubating cells with overexpressed $\mathrm{G}_{8} \mathrm{P}_{\mathrm{PD}}{ }^{31}$. Hence, in the present study we transfected $\mathrm{G} 8 \mathrm{P}_{\mathrm{PD}}$ plasmids at $24 \mathrm{~h}$ prior to the transfection of $\mathrm{CBE}^{17}$, sgRNA and EGFP-Y66C plasmids (Supplementary Fig. 1c). It was found that A3A CBE targeting of EGFPY66C reporter plasmid could recover the fluorescence in more than 30\% HEK293T cells (Fig. 1b and Supplementary Fig. 1d). Pre-incubation of cells with M13 and f1 G8P PD $_{\text {overexpression plasmid reduced }}$ fluorescent cells to approximately $10 \%$ of the whole population (Fig. 1b and Supplementary Fig. 1d), which confirmed our hypothesis that M13 and $\mathrm{f1}$ G8P $\mathrm{P}_{\mathrm{PD}}$ could also function as inhibitors to $\mathrm{BE}$. $\mathrm{G} 8 \mathrm{P}_{\mathrm{PD}}-\mathrm{free}$ pcDNA3.1 vector was transfected into HEK293T cells at $24 \mathrm{~h}$ prior to A3A CBE and sgRNA transfection to control for cell stress induced by serial transfections. Sanger sequencing of the PCR amplification product of A3A CBE-targeted site revealed evident C-to-T mutations in EGFP reporter (Fig. 1c and Supplementary Fig. 
1e), consistently suggesting that M13 and f1 G8P $P_{P D}$ could inhibit CBE activity in human cells. It appeared that

f1 G8P PD has significantly higher inhibitory activity than M13 G8P PD (Fig. 1b-c). Thus, subsequent studies are performed with f1 G8P $\mathrm{PD}$.

\section{G8PPD inhibits CBE targeting at endogenous genomic sites in human cells}

To examine the inhibitory effects of $\mathrm{G} 8 \mathrm{P}_{\mathrm{PD}}$ on $\mathrm{CBE}$ at endogenous sites, we designed sgRNA targeted to human EPPK1, GATAD2A, DNMT3B and DDX53 genes respectively for A3A CBE (Fig. 1d). Similar to the procedure in EGFP reporter assay, f1 G8P PD-encoding plasmid was transfected into HEK293T cells at $24 \mathrm{~h}$ prior to $\mathrm{A} 3 \mathrm{~A} \mathrm{CBE}$ and sgRNA transfection (Fig. 1e). The sgRNA-encoding and $\mathrm{fl}$ G8P $\mathrm{PD}_{\mathrm{PD}}$-encoding plasmids carried GFP and mCherry reporter genes respectively. In order to enrich cells with transfected plasmids, we used flow cytometry to enrich GFP and mCherry-dual positive cells (Supplementary Fig. 2) for analyses of CBE-induced genomic mutations.

Next-generation sequencing (NGS) analyses of sorted HEK293T cells revealed efficient base editing by A3A CBE at the four selected genomic sites (Supplementary Fig. 3a). The presence of f1 G8P PD reduced CBEmediated C-to-T conversion by various degree across different positions and genomic loci (Supplementary Fig. 3a). Statistical analyses of the editing efficiency at all examined positions revealed significant inhibitory activity of G8P PD toward A3A CBE (Fig. 1f). Comparing different target sites or sgRNA, we found that the averaged inhibition rates of each target site had no significant difference (Supplementary Fig. 3b), suggesting that the CBE-inhibiting activity of G8PPD was not dependent on genomic loci or sgRNA.

To investigate whether the activity of G8P $\mathrm{PD}_{\mathrm{PD}}$ was cell-type dependent, we examined the performance of G8P PD in U-2 OS cells. It was found that G8P PD reduced the editing efficiency of A3A CBE by various degree across different genomic sites and editing positions (Supplementary Fig. 4a) in U-2 OS. Similar to the results in HEK293T cells, the inhibitory activity of G8P $\mathrm{PD}_{\mathrm{PD}}$ in U-2 OS cells was not dependent on genomic loci or 
sgRNA (Supplementary Fig.4b). Collectively, these results suggested that G8P $P_{P D}$ could inhibit the editing activity of A3A CBE at different genomic sites across different cell types.

\section{G8PPD differentially inhibits the on-target and out-of-window editing of A3A CBE}

We next analyzed the inhibitory activity of G8P $P_{P D}$ at different editing positions within each target site. It was found that the inhibitory effects of $\mathrm{G} 8 \mathrm{P}_{\mathrm{PD}}$ displayed notable variations along the 20-bp targeting site for each sgRNA. This result was consistently observed in HEK293T and U-2 OS cells (Fig. 2a). This observation prompted us to compare the effects of G8P $\mathrm{P}_{\mathrm{PD}}$ on the editing positions 4 to 8 , which are conventionally deemed as the on-target editing window of CBEs, and on the out-of-window editing positions 1 to 3 and 9 to 20 . It was found that G8PPD significantly inhibited both the on-target and out-of-window editing of $\mathrm{A} 3 \mathrm{~A} \mathrm{CBE}$ in HEK293T cells, with the latter being reduced to minimum level (Fig. 2b). Interestingly, G8P $\mathrm{PD}_{\mathrm{P}}$ had minor or little inhibition toward the on-target editing of A3 A CBE but significantly inhibited the out-of-window editing (Fig. 2b).

The differential effects of G8P $\mathrm{PD}$ at the on-target and out-of-window editing positions were more evident when the inhibition rates were plotted along the 20-bp targeting site (Fig. 2c). Importantly, lowest inhibition rates were found at positions 4 to 8 , the canonical on-target positions of CBEs (Fig. 2b). The hot spots of G8P PD-mediated inhibition had significant overlap with the canonical out-of-window editing positions of CBEs (Fig. 2d). Comparative analyses of the inhibition of C-to-T conversion showed that G8P $\mathrm{PD}_{\mathrm{PD}}$ exhibited 2and 10-fold selectivity of inhibition toward out-of-window positions over on-target positions in HEK293T and U-2 OS cells respectively (Fig. 2e).

\section{G8PPD differentially inhibits the on-target and out-of-window editing of BE3 CBE and ABE7.10}

Because G8P $\mathrm{P}_{\mathrm{PD}}$ acts as a SpCas9 inhibitor, we envisioned that G8P $\mathrm{PD}_{\mathrm{P}}$ could inhibit different CBEs carrying 
SpCas9 module as the DNA-binding domain. Hence, we sought to examine the effects of G8P $\mathrm{PD}$ on BE3 CBE and ABE7.10 that contain deaminase domains different from that in $\mathrm{A} 3 \mathrm{~A} \mathrm{CBE}$. We found that $\mathrm{f} 1 \mathrm{G} 8 \mathrm{P}$ PD could suppress BE3 CBE-induced C-to-T conversion though the inhibitory effects appeared to be less prominent than that with A3A CBE (Supplementary Fig. 5a). Similar to the results with A3A CBE, the inhibitory effects of f1 G8P PD displayed varied inhibition rates at different editing positions of BE3 CBE (Fig. 3a), with the ontarget positions 4 to 8 exhibiting lower inhibition compared to the out-of-window positions (Fig. 3b). It was observed that G8P $\mathrm{PD}$ had 50-fold selectivity of inhibition toward the out-of-window positions over on-target positions (Fig. 3c).

We next examined the effects of G8P $\mathrm{PD}$ on ABE7.10. We designed four sgRNAs targeting to different genomic sites (Fig. 3d). Similar to the results with A3A and BE3 CBEs, f1 G8P PD inhibited the A-to-G conversion activity of ABE7.10 in a position-dependent manner with positions 4 to 7 , which is conventionally deemed as optimal on-target positions ${ }^{13}$, exhibiting lowest inhibition rates (Fig. 3e). f1 G8P PD showed 20-fold selectivity of inhibition toward the out-of-window positions over on-target positions of ABE7.10 (Fig. 3f). These results collectively demonstrated that G8P $\mathrm{PD}$ could preferentially inhibit the editing activities of CBE and $\mathrm{ABE}$ at out-of-window positions, suggesting the potential application of $\mathrm{G} 8 \mathrm{P}_{\mathrm{PD}}$ as an agent to improve the targeting scope of BEs.

As previous studies suggested that timed delivery of Acrs could improve the genome-editing specificity of SpCas $9^{30}$, we next sought to investigate the effects of AcrIIA4 on modulating CBE and ABE activities. HEK293T cells were co-transfected with AcrIIA4 plasmid and sgRNA- and BE-coding plasmids (Supplementary Fig. 6a) and the inhibitory activity of AcrIIA4 was examined. It was found that AcrIIA4 suppressed the on-target and out-of-window activities of ABE and CBE to undetectable levels (Supplementary Fig. 6b) and that the strong inhibitory activities were observed across all positions within each sgRNA or genomic site (Supplementary Fig. 6c-d). 


\section{G8PPD mediates precision correction of pathogenic $F B N 1$ mutation by CBEs}

To explore the potential therapeutic application of G8P $\mathrm{P}_{\mathrm{PD}}$, we assessed the effects of G8P $\mathrm{P}_{\mathrm{PD}}$ on the editing activities of A3A and BE3 CBEs in a cell-based disease model of Marfan syndrome ${ }^{32}$. In this cell model, a T7498C mutation was introduced into the FBN1 gene of HEK293T cells to model the pathogenic amino acid mutation $\mathrm{C}_{2500 \mathrm{R}^{32}}$. To enable CBE-mediated gene correction, we first designed sgRNA for A3A and BE3 CBEs to convert the pathogenic mutation T7498C to wild type (Fig. 4a). HEK293T-FBN1 ${ }^{\mathrm{T} 7498 \mathrm{C}}$ cells were transfected with A3A and BE3 CBEs respectively in the absence or presence of G8PPD. Sanger sequencing of the PCR amplification products of FBNl gene from edited cells revealed efficient CBE-induced C-to-T conversion (Fig. 4b). NGS analysis showed that A3A and BE3 CBEs induced C-to-T conversion with varied frequencies across the 20-bp target site (Fig. 4c). Similar to the previous results, G8PPD displayed remarkably higher inhibition rates at out-of-window positions than at on-target positions (Fig. 4d), with 36- and 3-fold selectivity for A3A and BE3 CBEs respectively (Fig. 4e). Most importantly, G8PPD reduced the frequency and the number of genotypes of incorrectly edited alleles of A3A and BE3 CBEs (Supplementary Figs.7-8) and increased perfectly edited alleles of A3A CBE from less than $4 \%$ to more than $38 \%$ of the whole population, the latter of which corresponds to more than $50 \%$ of the total edited alleles (Fig. 4f). This 9-fold improvement demonstrated the feasibility of using G8P $\mathrm{PD}$ as an agent for precision gene correction in Marfan syndrome model.

We also investigated the effects of G8P $\mathrm{PD}$ on high-fidelity CBE variants for correction of $F B N 1^{\mathrm{T} 7498 \mathrm{C}}$ mutation. It was found that $\mathrm{G} 8 \mathrm{P}_{\mathrm{PD}}$ could modestly inhibit the out-of-window editing activity of hA3A-eBEY130F at $\mathrm{C} 1$ position without affecting its on-targeting activity at $\mathrm{C} 4$ position (Fig. 4g). For BE3-R33A that exhibited only on-target editing at $\mathrm{C} 4$ position, G8P PD did not significantly affect its activity. These results together suggested that peptide inhibitors of SpCas9 such as G8P $\mathrm{PD}_{\mathrm{PD}}$ are compatible with and may even improve 
bioRxiv preprint doi: https://doi.org/10.1101/2020.11.02.364430; this version posted November 2, 2020. The copyright holder for this preprint (which was not certified by peer review) is the author/funder, who has granted bioRxiv a license to display the preprint in perpetuity. It is made available under aCC-BY-NC-ND 4.0 International license.

high-fidelity BE variants for precision gene correction. 


\section{Discussion}

It has well documented that CRISPR-Cas systems are associated with off-target cleavage ${ }^{33}$, chromosomal rearrangement ${ }^{34}$ and genotoxicity ${ }^{35}$. These adverse effects may result from uncontrolled expression of Cas proteins $^{22,36,37}$. Unfortunately, most therapeutically relavant applications of CRISPR-Cas involve constitutively active Cas nucleases that raises major safety concern ${ }^{38}$. With the increasing number of therapeutic applications of CRISPR-Cas, the ability to manipulate the activity of CRISPR-Cas in human cells has become urgently needed. One feasible approach is to develop CRISPR-Cas off-switches to control the intracellular activity of Cas proteins. Thus far, synthetic oligonucleotides ${ }^{28}$, small-molecule inhibitors ${ }^{29}$ and bacteriophage-derived anti-CRISPR proteins (Acrs) $)^{31}$ have been developed to enable the temporal control of CRISPR-Cas activity. It has been shown that Acrs can improve the targeting specificity of SpCas9 at on-target sites over off-target $\operatorname{sites}^{30}$. This improvement was achieved via precisely controlled, timed delivery of Acrs along with Cas9-sgRNA ribonucleoproteins (RNPs) ${ }^{30}$. Yet, the effects of Acrs on base editors have not been explored. Notably, small-molecule inhibitors of SpCas9 have been employed as inhibitors to Cas9-based transcription factors and base editors ${ }^{29}$.

In our previous study, we discovered that the in vitro and cellular activity of SpCas9 can be inhibited by the periplasmic domain of bacteriophage major coat proteins $\left(\mathrm{G} 8 \mathrm{P}_{\mathrm{PD}}\right)^{31}$. These G8P PD peptides disrupt Cas9sgRNA assembly by binding to the PAM interacting (PI) domain of SpCas $9^{31}$. It was found that time-controlled delivery of overexpressed G8P $\mathrm{PD}_{\mathrm{PD}}$ could improve the specificity of SpCas9 in human cells ${ }^{31}$. In the present study, we hypothesized that the out-of-window base editing of CBE may be attributed to excess CBE fusion proteins. Hence, we sought to explore whether timed delivery of SpCas9-inactivating G8P $\mathrm{PD}$ could inhibit and improve the activities of $\mathrm{CBE}$ and $\mathrm{ABE}$, in a rationale similar to that with the genome editing of $\operatorname{SpCas} 9^{31}$. Our results have demonstrated that G8P $\mathrm{PD}$ can preferentially inhibit the out-of-window editing of $\mathrm{A} 3 \mathrm{~A}$ and BE3 CBEs and ABE7.10, leading to more focused targeting window. To the best of our knowledge, our study 
represents the first peptide inhibitors that can improve the targeting scope of BEs. In comparison with G8PD, AcrIIA4 abolished the activities of $\mathrm{CBE}$ and $\mathrm{ABE}$ at both on-target and out-of-window sites without any selectivity. These results support the notion that weak inhibitors, rather than strong inhibitors, are optimal agents for modulating the specificity of genome-editing tools. Nevertheless, it is possible that the editing activity of BEs may be improved by limiting the exposure time of SpCas9 to Acrs where Acrs are delivered at later time points during transfection.

It has been reported that the targeting specificity of CBEs and ABEs can be improved by incorporating genetic mutations into deaminase domain ${ }^{20,21}$. These $\mathrm{BE}$ variants are extremely useful when precision base editing is needed for therapeutic applications. In the present study, we have shown that G8P $\mathrm{PD}_{\mathrm{PD}}$ has minor yet significant inhibition at the out-of-window editing position of hA3A-eBE-Y130F CBE. Most importantly, G8P $P_{P D}$ dose not affect the on-target editing activities of hA3A-eBE-Y130F and BE3-R33A CBEs. These results have established the feasibility of $\mathrm{G} 8 \mathrm{P}_{\mathrm{PD}}$ not only as an alternative approach to improving the targeting specificity of BEs but also as an additive agent to combine with BE variants. In further studies, it would be interesting to evaluate the inhibitory activities of G8P $\mathrm{PD}_{\mathrm{PD}}$ toward dual adenine and cytidine base editors (A\&C$\mathrm{BEs})^{39}$ and prime editors ${ }^{40}$. These studies can advance our understanding of the capability of G8P $\mathrm{P}_{\mathrm{PD}}$ serving as general modulators of CRISPR-Cas genome engineering tools.

Although we only examined f1 and M13 G8P PD for inhibition of CBE activity in the current study, there might be other G8P $P_{P D}$ from inoviridae phages ${ }^{31}$ that can function as CBE off-switches. Comparative analyses can be performed on these different G8P $\mathrm{PD}$ peptides to determine the sequence-activity relationship. This information would facilitate the design and development of next-generation peptide off-switches of CBEs. Moreover, in-depth understanding of the mechanism of action of G8P $P_{P D}$ on $\mathrm{CBE}$ inhibition can also advance their therapeutic applications as modulators for precision gene correction. 


\section{Methods}

\section{Plasmid construction}

sgRNA was cloned into pGL3-sgRNA expression vector carrying a U6 promoter and an EGFP reporter gene (Addgene, \#107721). CBE-targeted genomic sites are indicated in Supplementary Table 1. The sequence of sgRNA-encoding oligonucleotides were listed in Supplementary Table 2. Human codon-optimized DNA sequences encoding M13 and f1 G8P $\mathrm{PD}_{\mathrm{PD}}$ were cloned into the BamH I/Xba I sites of pcDNA3.1(+) by plasmid recombination kit Clone Express (Vazyme Biotech, Nanjing, China). These G8P PD peptides carry a Cterminal SV40 nuclear localization signal (NLS) for co-localization with Cas9 proteins. G8P PD peptides were cloned into plv-EF1 $\alpha$-mCherry plasmid harboring mCherry fluorescent protein marker. For construction of EGFP-Y66C reporter plasmid, Y66C mutation was introduced by quickchange PCR.

\section{Cell culture and transfection}

HEK293T and U-2 OS cells were obtained from ATCC and maintained in Dulbecco's Modified Eagle Medium (DMEM) (Cat. No. SH30243, Hyclone, Logan, USA) supplemented with 10\% fetal bovine serum (FBS) and $1 \%$ penicillin-streptomycin at $37{ }^{\circ} \mathrm{C}$ with $5 \% \mathrm{CO}_{2}$. Transfection was performed using lipofectamine 2000 Reagent (Cat. No. 11668019, ThermoFisher Scientific, Waltham, USA) according to manufacturer's instructions. HEK293T and U-2 OS cells were seeded on to poly-D-lysine (Cat. No. A-003-E, Sigma, St. Louis, USA) pre-coated 24-well plates at $24 \mathrm{~h}$ prior to transfection.

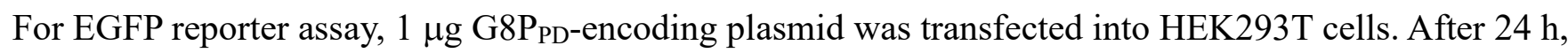
$0.25 \mu \mathrm{g}$ sgRNA plasmid, $0.5 \mu \mathrm{g}$ A3A CBE plasmid and $0.05 \mu \mathrm{g}$ the EGFP-Y66C reporter plasmid were cotransfected into $\mathrm{G} 8 \mathrm{P}_{\mathrm{PD}}$-expressing cells. At $48 \mathrm{~h}$ after the second transfection, cells were harvested and analyzed by flow cytometry. For base editing at endogenous genes, cells of approximately $70 \%$ confluency were transfected with $1 \mu \mathrm{g}$ G8P PD plasmid that carries an mCherry selection marker at $12 \mathrm{~h}$ after seeding. At 
$24 \mathrm{~h}$ after G8P $\mathrm{PD}_{\mathrm{P}}$ transfection, $0.25 \mu \mathrm{g}$ sgRNA plasmid that carries a GFP selection marker and $0.5 \mu \mathrm{g}$ CBE plasmid were transfected in G8P $\mathrm{PD}_{\mathrm{PD}}$-expressing cells. At $48 \mathrm{~h}$ after $\mathrm{CBE}$ and sgRNA plasmids transfection, cells were harvested and analyzed by Beckman Coulter CytFLEX (Beckman Coulter, Brea, USA) or sorted by BD FACSAria III flow cytometry (BD Biosciences, New York, USA). At least 2,000 mCherry and GFP dual positive cells were collected for subsequent analyses. Analyses of the Sanger sequencing results of the PCR product of edited EGFP reporter were performed using EditR ${ }^{41}$.

For AcrIIA4 co-transfection experiments, HEK293T cells were transfected with $0.5 \mu \mathrm{g}$ of CBE plasmid and $0.25 \mu \mathrm{g}$ sgRNA-expressing and $1 \mu \mathrm{g}$ of AcrIIA4-expressing plasmids that carry GFP and mCherry reporters, respectively. At $72 \mathrm{~h}$ after transfection, mCherry and GFP dual positive cells were collected for subsequent analyses.

\section{Extraction and PCR amplification of genomic DNA}

Genomic DNA was extracted using QuickExtract ${ }^{\mathrm{TM}}$ DNA Extraction Solution (Lucigen, USA). Genomic PCR was performed using $100 \mathrm{ng}$ genomic DNA, corresponding primers (Supplementary Table 3-4), Phanta Max Super-fidelity DNA Polymerase (Cat. No. P505-d1, Vazyme) or KOD plus (Cat. No. F0934K, Takara, Kyoto, Japan) with a touchdown cycling protocol that contains 30 cycles of $98{ }^{\circ} \mathrm{C}$ for $10 \mathrm{~s}, \mathrm{X}{ }^{\circ} \mathrm{C}$ for $15 \mathrm{~s}$ where $\mathrm{X}$ decreases from $68{ }^{\circ} \mathrm{C}$ to $58{ }^{\circ} \mathrm{C}$ with a $-1{ }^{\circ} \mathrm{C} /$ cycle rate and $68{ }^{\circ} \mathrm{C}$ for $60 \mathrm{~s}$.

\section{NGS analyses of PCR amplicons}

PCR was performed using the NGS primers (Supplementary Table 4). PCR products were purified using Gel Extraction Kit (Cat. No. D2500-02, OMEGA) before construction of NGS libraries. Hiseq3000 SBS\&Cluster high-throughput NGS library preparation kit (Cat. No. FC-410-1002, Illumina, San Diego, CA, USA), (VAHTS Universal DNA Library Prep Kit (ND608-01, Vazyme) or TruSeq NanoDNALT Library Prep Kit 
(Illumina) were used to generate dual-indexed sequence following the manufacturer's protocol. Briefly, more than $50 \mathrm{ng}$ purified PCR fragment was used for direct library preparation. The fragments were treated with End Prep Enzyme Mix (Cat. No. WE0229, Illumina) for end repairing, 5' phosphorylation and dA-tailing in one reaction, followed by a T-A ligation to add adaptors to both ends. Size selection of adaptor-ligated DNA was then performed using VAHTSTM DNA Clean beads (Cat. No. N411-03, Vazyme) or Beckman AMPure XP beads (Cat. No. A63882, Illumina). Each sample was then amplified by PCR for 8 cycles using P5 and P7 primers (Supplementary Table 4). Both P5 and P7 primers carry the sequences that can anneal with flow cells for bridge PCR. In addition, P7 primer carries a six-base index allowing for multiplexing. The PCR products were cleaned using VAHTSTM DNA Clean beads (Cat. No. N411-03, Vazyme) or Beckman AMPure XP beads (Cat. No. A63882, Illumina), validated using an Agilent 2100 Bioanalyzer (Agilent Technologies, Palo Alto, CA, USA) and quantified by Qubit2.0 Fluorometer (Invitrogen, Carlsbad, CA, USA) or Quant-iT PicoGreen dsDNA Assay Kit (ThermoFisher). Two or three biological replicates were processed by Genewiz (Suzhou, China) or Personalbio (Shanghai, China) using Illumina HiSeq 3000. Sequencing was carried out using a $2 \times 150$ paired-end $(\mathrm{PE})$ or $2 \times 300$ paired-end $(\mathrm{PE})$ configuration. Image analyses and base calling were conducted by the HiSeq Control Software (HCS) + OLB + GAPipeline-1.6 (Illumina) on the HiSeq instrument. Sequencing reads were obtained in the Fastq format. Amplicons with less than $6 \mathrm{M}$ read counts were excluded from the analyses.

To obtain the editing efficiencies, the adapter pair of the pair-end reads were removed using AdapterRemoval version 2.2.2, and pair end read alignments of $11 \mathrm{bp}$ or more bases were combined into a single consensus read. All processed reads were then mapped to the target sequences using the BWA-MEM algorithm (BWA v0.7.16). For each site, the mutation rate was calculated using bam-readcount with parameters -q 20 -b 30. Indels were calculated based on reads containing at least 1 inserted or deleted nucleotide in protospacer. Indel frequency was calculated as the number of indel-containing reads/total mapped reads. 


\section{Data availability}

High-throughput sequencing data has been deposited in the NCBI Sequence Read Archive database under the accession code PRJNA601083. Additional materials or information can be obtained from J.L. upon reasonable request.

\section{Statistical analyses}

For cell-based assay, three biological replicates are generally performed and the results are shown as mean \pm standard error of the mean (SEM). For NGS analyses, two or three biological replicates are performed. Statistical analyses were performed using two-tailed Student's $t$ test unless otherwise noted.

\section{Acknowledgements}

We thank the High-Throughput Screening Platform and Biomedical Big Data Platform at Shanghai Institute for Advanced Immunochemical Studies (SIAIS) at ShanghaiTech University for the support of flow cytometry experiments and analyses of NGS data. This work is supported by the National Natural Science Foundation of China (31600686 to J.L.) and ShanghaiTech University Startup Fund (2019F0301-000-01 to J.L.)

\section{Author contributions}

J.L. conceptualized study. J.L., K.J. and Y.-R.C. designed the experiments, S.H. analyzed next-generation sequencing data. K.J. and Y.-R.C. performed the in vivo CBE-inhibiting experiments. Z.L. and P.M. provided critical resources. J.L. and K.J. and Y.-R.C. wrote the manuscript. All authors discussed the results and approved the manuscript. 


\section{References}

1. Barrangou, R. et al. CRISPR provides acquired resistance against viruses in prokaryotes. Science 315, 1709-12 (2007).

2. Brouns, S. J. et al. Small CRISPR RNAs guide antiviral defense in prokaryotes. Science 321, 960-4 (2008).

3. Marraffini, L. A. \& Sontheimer, E. J. CRISPR interference limits horizontal gene transfer in staphylococci by targeting DNA. Science 322, 1843-5 (2008).

4. Pickar-Oliver, A. \& Gersbach, C. A. The next generation of CRISPR-Cas technologies and applications. Nat. Rev. Mol. Cell Biol. 20, 490-507 (2019).

5. Jinek, M. et al. RNA-programmed genome editing in human cells. Elife 2, e00471 (2013).

6. Mali, P. et al. RNA-guided human genome engineering via Cas9. Science 339, 823-6 (2013).

7. Cong, L. et al. Multiplex genome engineering using CRISPR/Cas systems. Science 339, 819-23 (2013).

8. Cho, S. W., Kim, S., Kim, J. M. \& Kim, J. S. Targeted genome engineering in human cells with the Cas9 RNA-guided endonuclease. Nat. Biotechnol. 31, 230-2 (2013).

9. Liang, P. et al. Correction of beta-thalassemia mutant by base editor in human embryos. Protein Cell 8, 811-822 (2017).

10. Villiger, L. et al. Treatment of a metabolic liver disease by in vivo genome base editing in adult mice. Nat Med 24, 1519-1525 (2018).

11. Zeng, J. et al. Therapeutic base editing of human hematopoietic stem cells. Nat Med 26, 535-541 (2020).

12. Komor, A. C., Kim, Y. B., Packer, M. S., Zuris, J. A. \& Liu, D. R. Programmable editing of a target base in genomic DNA without double-stranded DNA cleavage. Nature 533, 420-4 (2016).

13. Gaudelli, N. M. et al. Programmable base editing of $A^{*} T$ to $G^{*} \mathrm{C}$ in genomic DNA without DNA cleavage. Nature 551, 464-471 (2017). 
14. Rees, H. A. \& Liu, D. R. Base editing: precision chemistry on the genome and transcriptome of living cells. Nat. Rev. Genet. 19, 770-788 (2018).

15. Cox, D. B. T. et al. RNA editing with CRISPR-Cas13. Science 358, 1019-1027 (2017).

16. Abudayyeh, O. O. et al. A cytosine deaminase for programmable single-base RNA editing. Science 365, 382-386 (2019).

17. Wang, X. et al. Efficient base editing in methylated regions with a human APOBEC3A-Cas9 fusion. Nat. Biotechnol. 36, 946-949 (2018).

18. Kim, Y. B. et al. Increasing the genome-targeting scope and precision of base editing with engineered Cas9-cytidine deaminase fusions. Nat. Biotechnol. 35, 371-376 (2017).

19. Gehrke, J. M. et al. An APOBEC3A-Cas9 base editor with minimized bystander and off-target activities. Nat. Biotechnol. 36, 977-982 (2018).

20. Jin, S. et al. Cytosine, but not adenine, base editors induce genome-wide off-target mutations in rice. Science 364, 292-295 (2019).

21. Zuo, E. et al. Cytosine base editor generates substantial off-target single-nucleotide variants in mouse embryos. Science 364, 289-292 (2019).

22. Hsu, P. D. et al. DNA targeting specificity of RNA-guided Cas9 nucleases. Nat. Biotechnol. 31, 82732 (2013).

23. Kim, S., Kim, D., Cho, S. W., Kim, J. \& Kim, J. S. Highly efficient RNA-guided genome editing in human cells via delivery of purified Cas9 ribonucleoproteins. Genome Res. 24, 1012-9 (2014).

24. Rees, H. A. et al. Improving the DNA specificity and applicability of base editing through protein engineering and protein delivery. Nat. Commun. 8, 15790 (2017).

25. Bondy-Denomy, J., Pawluk, A., Maxwell, K. L. \& Davidson, A. R. Bacteriophage genes that inactivate the CRISPR/Cas bacterial immune system. Nature 493, 429-32 (2013). 
26. Pawluk, A., Davidson, A. R. \& Maxwell, K. L. Anti-CRISPR: discovery, mechanism and function. Nat. Rev. Microbiol. 16, 12-17 (2018).

27. Stanley, S. Y. \& Maxwell, K. L. Phage-encoded anti-CRISPR defenses. Annu. Rev. Genet. 52, 445-464 (2018).

28. Li, B. et al. Synthetic oligonucleotides inhibit CRISPR-Cpfl-mediated genome editing. Cell Rep. 25, 3262-3272 e3 (2018).

29. Maji, B. et al. A high-throughput platform to identify small-molecule inhibitors of CRISPR-Cas9. Cell 177, 1067-1079 (2019).

30. Shin, J. et al. Disabling Cas9 by an anti-CRISPR DNA mimic. Sci. Adv. 3, e1701620 (2017).

31. Cui, Y. R. et al. Allosteric inhibition of CRISPR-Cas9 by bacteriophage-derived peptides. Genome Biol. 21, 51 (2020).

32. Zeng, Y. et al. Correction of the Marfan syndrome pathogenic FBN1 mutation by base editing in human cells and heterozygous embryos. Mol. Ther. 26, 2631-2637 (2018).

33. Koo, T., Lee, J. \& Kim, J. S. Measuring and reducing off-target activities of programmable nucleases including CRISPR-Cas9. Mol. Cells 38, 475-81 (2015).

34. Kosicki, M., Tomberg, K. \& Bradley, A. Repair of double-strand breaks induced by CRISPR-Cas9 leads to large deletions and complex rearrangements. Nat. Biotechnol. 36, 765-771 (2018).

35. Li, C. et al. HDAd5/35(++) adenovirus vector expressing anti-CRISPR peptides decreases CRISPR/Cas9 toxicity in human hematopoietic stem cells. Mol. Ther. Methods Clin. Dev. 9, 390-401 (2018).

36. Pattanayak, V. et al. High-throughput profiling of off-target DNA cleavage reveals RNA-programmed Cas9 nuclease specificity. Nat. Biotechnol. 31, 839-43 (2013).

37. Fu, Y., Sander, J. D., Reyon, D., Cascio, V. M. \& Joung, J. K. Improving CRISPR-Cas nuclease 
specificity using truncated guide RNAs. Nat. Biotechnol. 32, 279-84 (2014).

38. Fellmann, C., Gowen, B. G., Lin, P. C., Doudna, J. A. \& Corn, J. E. Cornerstones of CRISPR-Cas in drug discovery and therapy. Nat. Rev. Drug Discov. 16, 89-100 (2017).

39. Zhang, X. H. et al. Dual base editor catalyzes both cytosine and adenine base conversions in human cells. Nat. Biotechnol. 38, 856-U10 (2020).

40. Anzalone, A. V. et al. Search-and-replace genome editing without double-strand breaks or donor DNA. Nature 576, 149-+ (2019).

41. Kluesner, M. G. et al. EditR: A method to quantify base editing from Sanger sequencing. CRISPR J. 1, 239-250 (2018). 


\section{Figures}
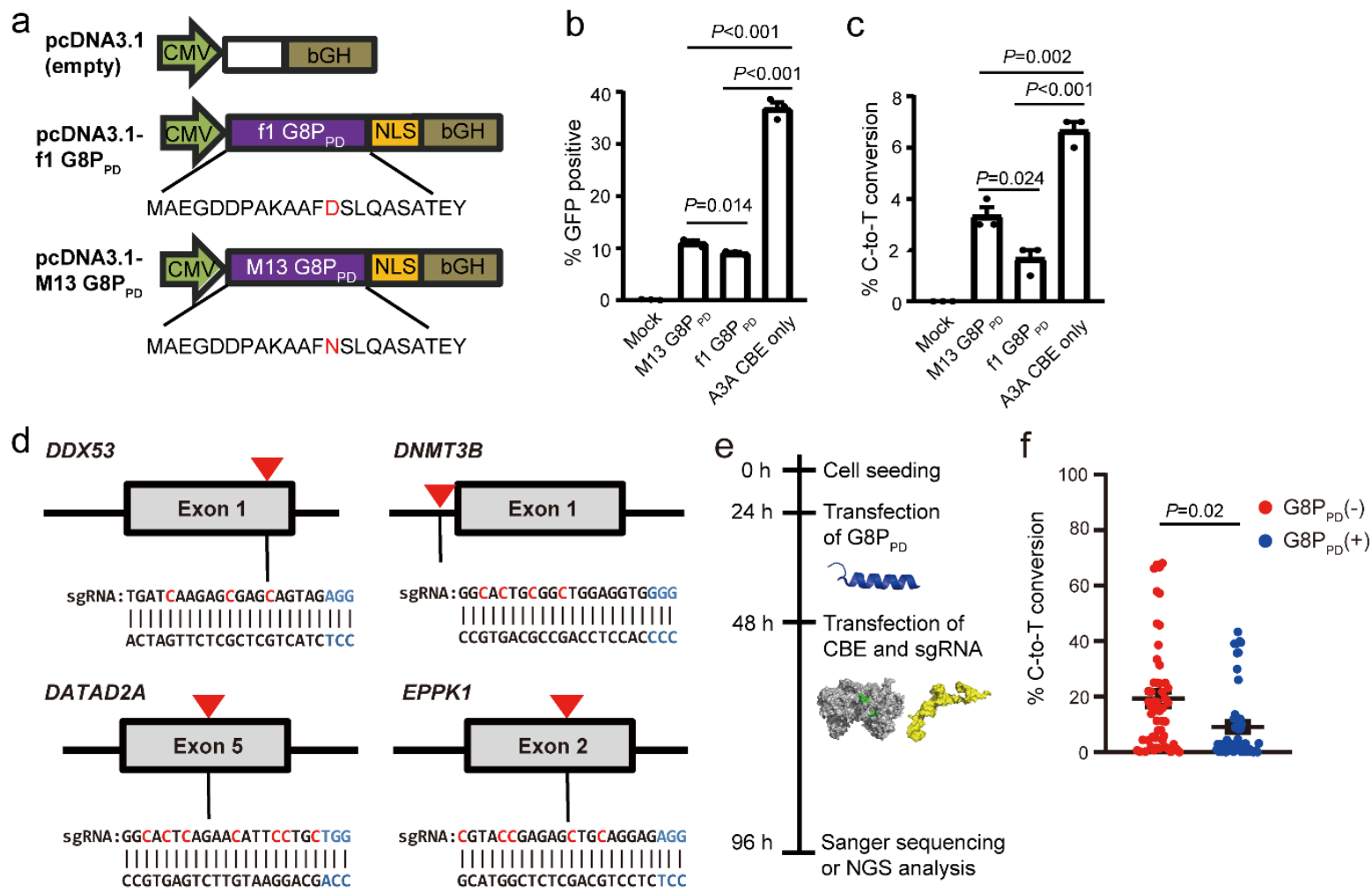

Fig. 1: G8Ppo peptides inhibit CBE targeting of EGFP reporter and endogenous genomic sites in human

cells. a The design of G8PDD plasmids for mammalian expression. c Flow chart showing experimental procedures. b-c The inhibitory effects of M13 and f1 G8P $\mathrm{PD}$ toward A3A CBE in EGFP reporter cells, as determined by Sanger sequencing. d Design of sgRNA targeted to DDX53, DNMT3B, GATAD2A and EPPKI genomic loci. The PAM sequence and cytidines are highlighted in blue and red, respectively. Red arrows denote the positions of selected target sites. e Flow chart showing experimental procedures. The cartoons are blue for G8P $\mathrm{PD}_{\mathrm{PD}}$, grey for $\mathrm{CBE}$ and yellow for sgRNA, respectively. $\mathbf{f}$ Analysis of the frequency of C-to-T conversion at all editing positions in the absence and presence of G8P PD. Significant difference between the groups with and without f1 G8P PD is determined using two-tailed Student's $t$ test. 
a

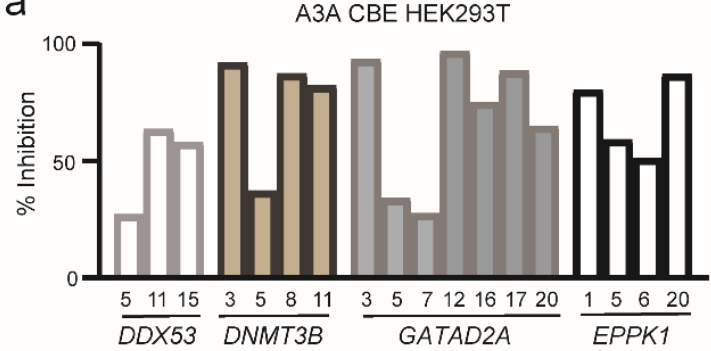

b
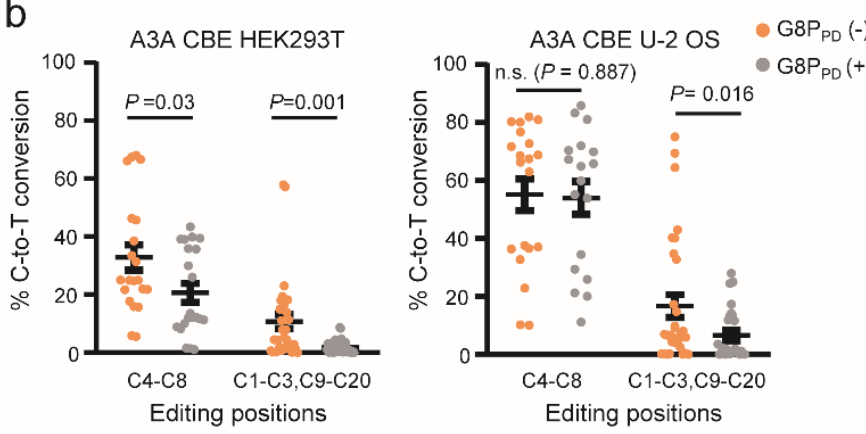

d 1

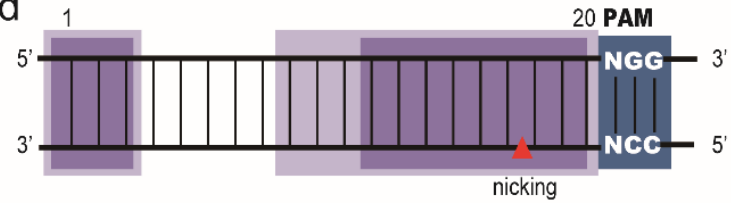

A3A CBE U-2 OS

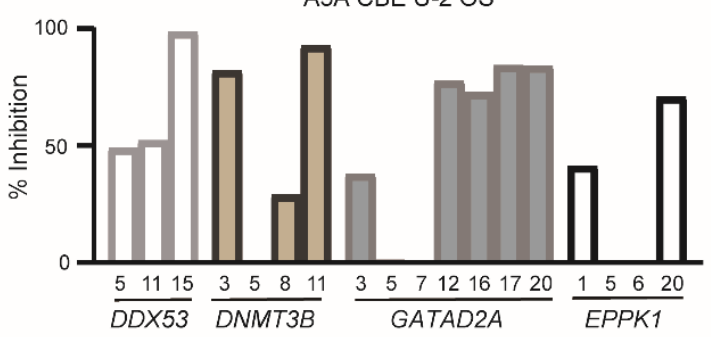

C

A3A CBE editing positions

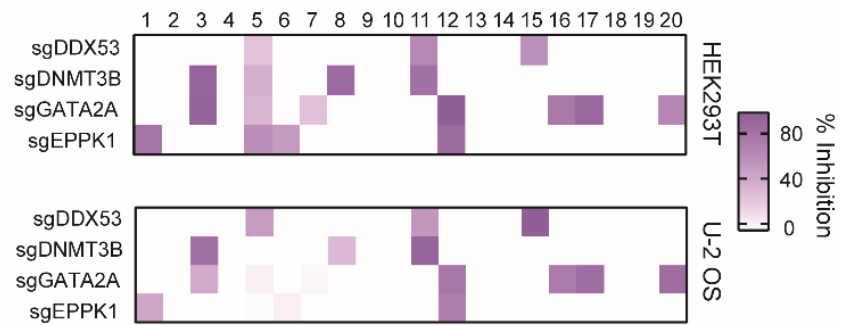

e

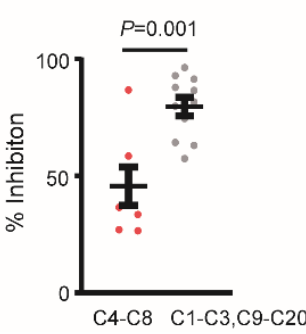

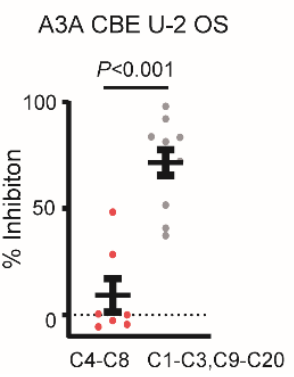

Fig. 2: The inhibitory activities of f1 G8PPD toward A3A CBE at the endogenous genomic sites in human

cell lines. a The inhibitory activity of f1 G8P PD toward A3A CBE in HEK293T and U-2 OS cells. b Positiondependent inhibitory activity of G8P $\mathrm{PD}$ at on-target and out-of-window editing positions of A3A CBE. Significant difference is determined using two-tailed Student's $t$ test. n.s., not significant. $\mathbf{c}$ Heat map showing position-dependent inhibition of A3A CBE by G8P $\mathrm{PD}$. d Schematic presentation of the canonical out-ofwindow position (light purple) and the hot spots of G8PPD inhibition (dark purple). PAM sequences are highlighted in blue. e Comparison of the inhibition rates of $\mathrm{G} 8 \mathrm{P}_{\mathrm{PD}}$ at the on-target and out-of-window editing positions of $\mathrm{A} 3 \mathrm{~A} \mathrm{CBE}$. 
a

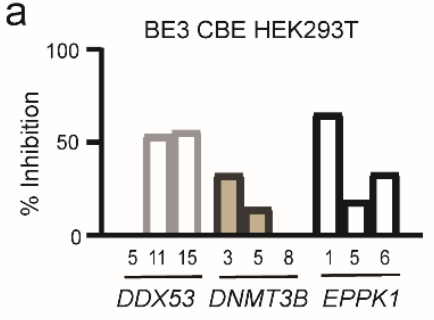

d

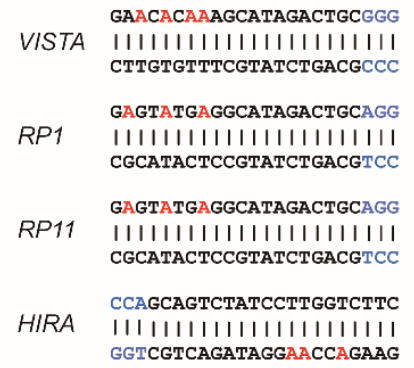

b

BE3 CBE editing positions

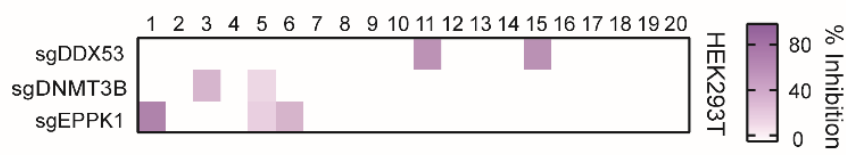

e

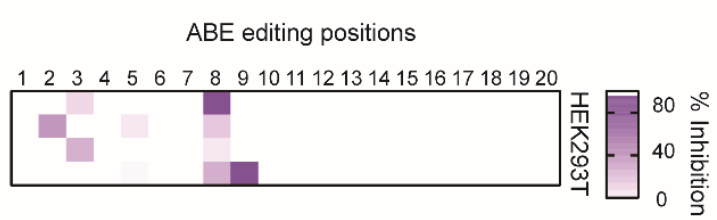

C BE3 CBE HEK293T

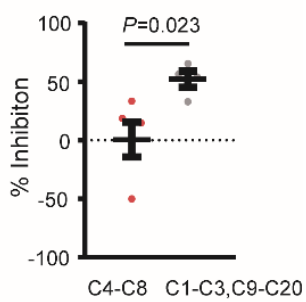

f

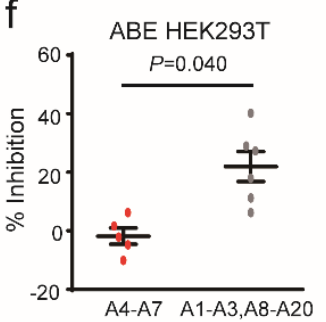

Fig. 3: The inhibitory activities of f1 G8PPD toward BE3 CBE and ABE7.10 at the endogenous genomic

sites in human cell lines. a The inhibitory activity of $\mathrm{f1}$ G8P $\mathrm{PD}$ toward BE3 CBE in HEK293T cells. b Heat map showing position-dependent inhibition of $\mathrm{BE} 3 \mathrm{CBE}$ by G8PD. $\mathbf{c}$ Comparison of the inhibition rates of G8P $\mathrm{PD}$ at the on-target and out-of-window editing positions of BE3 CBE. d Design of sgRNA for ABE7.10 targeted genomic loci. The PAM sequence and adenine positions are highlighted in blue and red, respectively. e Heat map showing the position-dependent inhibition of ABE7.10 by G8P PD. $\mathbf{f}$ Comparison of the inhibition rates of G8P $P_{P D}$ at the on-target and out-of-window editing positions of ABE7.10. 


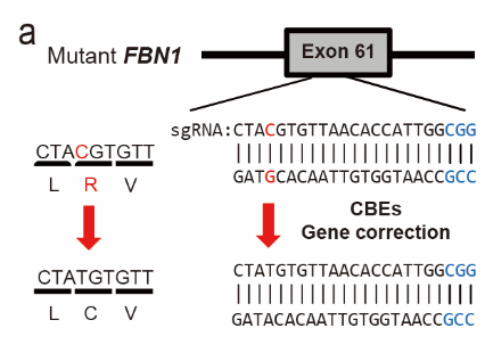

b
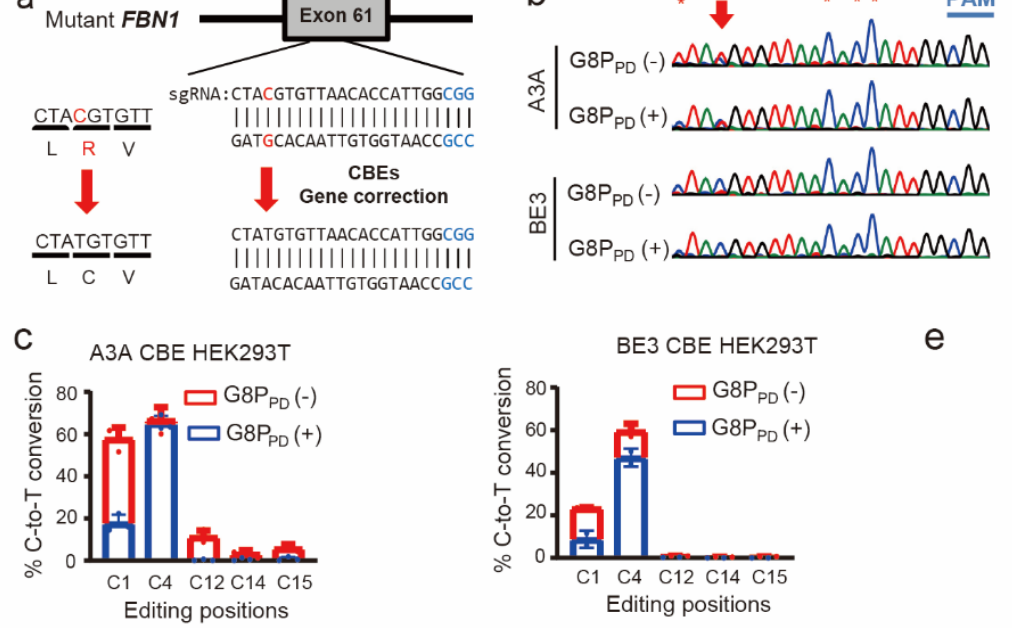

f

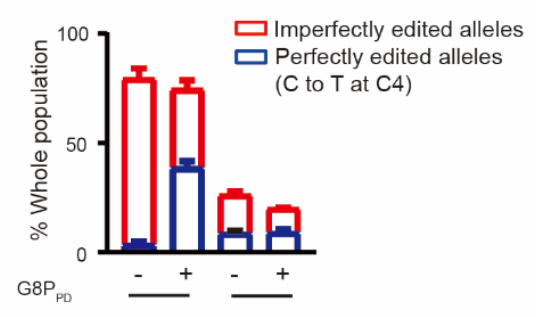

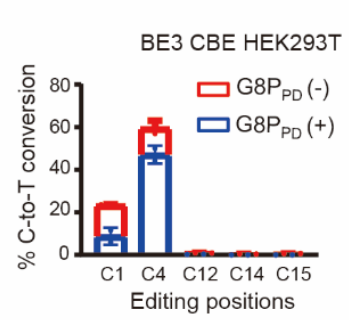

g

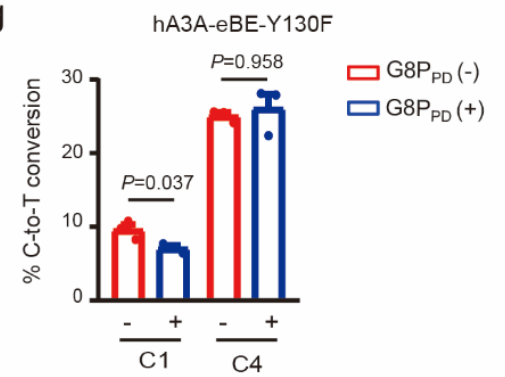

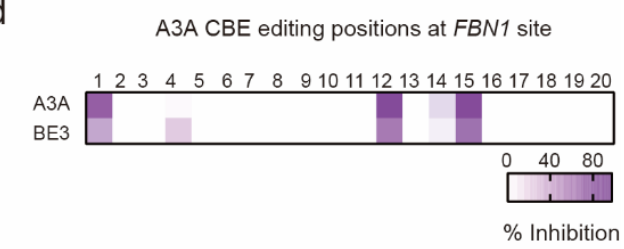

e

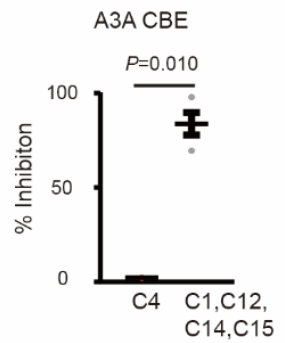

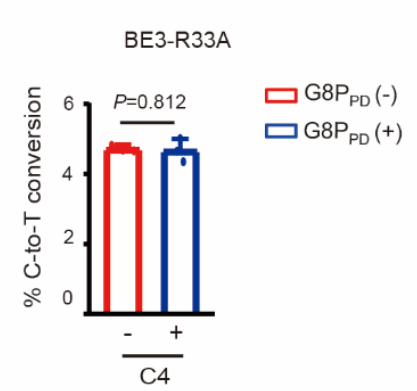

Fig. 4: G8PPD improves the targeting window of CBEs for correction of $F B N 1^{T 7498 C}$ mutation. a

Schematic illustration of sgRNA design and the strategy for CBE-based gene correction. b Sanger sequencing of the positive strand of FBN1 illustrating CBE-induced C-to-T mutations. Arrows and asterisks denote ontarget and out-of-window cytidine positions for base editing. $\mathbf{c}$ Frequency of A3A and BE3 CBE-mediated Cto- $\mathrm{T}$ conversion in the absence and presence of f1 G8P $\mathrm{PD}_{\mathrm{P}}$ d Heat map showing the position-dependent inhibition of $\mathrm{A} 3 \mathrm{~A}$ and $\mathrm{BE} 3 \mathrm{CBEs}$ by G8P $\mathrm{PD}$. e Comparison of the inhibition rates of G8P $\mathrm{PD}$ at the on-target and out-of-window editing positions of A3A and BE3 CBEs at FBN1 site. Significant difference is determined using Student's $t$ test. f Analyses of perfectly edited FBN1 alleles in the absence and presence of G8PPD. Unless noted otherwise, the data are shown as mean $\pm \operatorname{SEM}(n=2$ or 3$)$ and significant difference is determined using two-tailed Student's $t$ test. g The effects of f1 G8P correction of $F B N 1^{\mathrm{T} 7498 \mathrm{C}}$ mutation. 